\title{
Uniform Convergence of the Series Expansion of the Multifractional Brownian Motion
}

\author{
BA Demba Bocar
}

UFR SET Thies of University Senegal, Thies, Senegal

Email address:

dbba@univ-thies.sn

\section{To cite this article:}

BA Demba Bocar. Uniform Convergence of the Series Expansion of the Multifractional Brownian Motion. Applied and Computational Mathematics. Vol. 9, No. 6, 2020, pp. 195-200. doi: 10.11648/j.acm.20200906.14

Received: June 17, 2020; Accepted: October 16, 2020; Published: December 4, 2020

\begin{abstract}
In this paper we define the multifractional Brownian motion and we give some properties. we study the uniform Convergence of the Serie expansion. After having determined the covariance function, we give in proposition 2 another proof of almost sure uniform convergence on compact $\mathrm{K}$ of the series. We will finish by showing that the m.B.f is locally astymptotically self-similar, with field or fractional Brownian field with Hurst exposant H. One of the problem, for application of multifractional Brownian motion, is the regularity of the function. In the filtered white noise model the increments are no more homogeneous as in fractional Brownian field case. It is obvious when we consider the tangent field associated with a function. Still the multifractional function in the previous model is constant and it is not convient for many applications. We show the uniform convergence of the series on $\mathrm{K}$. We deduce from the previous questions the almost sure uniform convergence of the series to a $\mathrm{mBm}$.
\end{abstract}

Keywords: Multifractional Brownian Motion, Uniform Convergence, Series Expansion

\section{Introduction}

The multifractional Brownian motion (mBf) are Gaussian processes which exhibit local-similary. They have been introduced in the seminal papers of R.F Peltier and LevyVehel, Benassi and as extensions to theclass of fractional Brownian motion(fBm) processes [14].

This natural extension of $\mathrm{fBm}$ results in some sense in a loss of properties the increments of $\mathrm{mBm}$ indeed are non stationary and the process is no more self-similar.

One generalize the definition of the fractional Brownian motion of exponent $\mathrm{H}$ to the case where $\mathrm{H}$ is no longer a constant, but a function of the time index of the processes.

The new process does provide a useful model for host of continuous and non stationary natural signals. It is wells Know that fBm's are self-similar processes allowing to conveniently describe irregular signals which arise in many situations [7], [4] and [12].

However, the pointwise irregularity of an $\mathrm{fBm}$ is the same all along its path.

This last property is sometimes undesirable, since it restricts the fields of application. For instance, fBm have frequently been used for synthesizing artificial mountains.

Such a modeling assumes that the irregularity of a mountain is everywhere the same. It appears that in reality this assumption is too strong because in particular, one does not take into account erosion phenonena.

Consequently, it should be convenient to relax the contraint of stationarity and be instead able to control the local irregularity.

The $\mathrm{mBm}$ seems to be the simplest generalisation of $\mathrm{fBm}$ that fulfills this requirement.

Let us gives two examples. The long term correlation of the increments of $\mathrm{fBm}$ decay as $K^{2 H-2}$, resulting in long range dependence when $H>\frac{1}{2}$ and anti-persistent behavior when $H<\frac{1}{2}$. Also, almost surly, for eacht its pointwise Hölder exponent is equal to $\mathrm{H}$. Since $\mathrm{H}$ rules both ends of the Fourier spectrum, i.e the high frequencies related to the Hölder regularity and the low frequencies related to the long term dependence structure, it is not possible to have at the same time a very irregular local behavior and long range dependence (implying $H>\frac{1}{2}$ ).

As a consequence, $\mathrm{fBm}$ is not adapted to model phenomena which display both there features, such as Internet traffic or 
certain highly textured images with strong global organisation. Another example is in the field of image synthesis: $\mathrm{fBm}$ has frequently been used for generating artificial mountains. Such a modeling assumes that the regularity of the mountain is everywhere the same. This is not realistic, since it does not take into account erosion or other meteorological phenomena which smooth some parts of mountains more than others.

Multifractional Brownian motion was introduced to overcome there limitations. The basic idea is to replace the real $\mathrm{H}$ by a function $\mathrm{h}(\mathrm{t})$.

\section{Preliminaries}

Definition 2.1. Let $\left(X, d_{X}\right),\left(Y, d_{Y}\right)$ be two metric spaces. A function $f: X \mapsto Y$ is called a Hölder function of exponent $\beta>0$, if for each $\mathrm{x}, \mathrm{y} \in \mathrm{X}$ such that $d_{X}(x, y)<1$ we have

$$
d_{Y}(f(x), f(y)) \leq C d_{X}(x, y)^{\beta} .
$$

Definition 2.2. Let $H:[0,+\infty) \mapsto(0,1)$ be a Hölder function of exponent $\beta>0$. For $t \geq 0$ the following random function, denoted by $W_{H}(t)$ or $W_{H}(t)$ is called reduced multifractional Brownian motion with functional parameter $\mathrm{H}$ :

$$
W_{H_{t}}=\frac{1}{\Gamma\left(H_{t}+\frac{1}{2}\right)}\left\{\int_{-\infty}^{0}\left[(t-s)^{H_{t}-\frac{1}{2}}-(-s)^{H_{t}-\frac{1}{2}}\right] d W(s)+\int_{0}^{t}(t-s)^{H_{t}-\frac{1}{2}} d W(s)\right\}
$$

where $\mathrm{W}$ denotes the ordinary Brownian motion and the integration is taken in the mean square sense.

The multifractional Brownian motion processes (MBM) are obtained by allowing the self-similarity parameter $\mathrm{H}$ of to depend on time.

This can be done by using various representations of the FBM processes.

R.F Peltier and Levy-Vehel [14] have introduced the MBM process

$$
Y_{(0,1)}(t)=\int_{R}\left((t-u)_{t}^{H(t)-\frac{1}{2}}-(-u)_{t}^{H(t)-\frac{1}{2}} W(d u)\right)
$$

where

$$
x_{t}^{\delta}=x^{\delta}, \quad x>0 \quad \text { and } \quad x_{t}^{\delta}=0, \quad x \leqslant 0 .
$$

Here the function $\mathrm{H}(\mathrm{t})$ takes values in the range $(0,1)$ and $\mathrm{W}(\mathrm{du})$ denotes an independently scattered standard Gaussian measure on $\mathbb{R}$.

The above integral is well-defined in the $\mathbb{L}^{2}$-sense since $0<H(t)<1$ and it can be viewed as an integral moving average type representation of the process $Y_{0,1}$.[7] and [11]

On the other hand, $\tilde{Y}=\{\tilde{Y}(t)\}_{t \in \mathbb{R}}$ based on a harmonizable integral representation of the fractional Brownian motion. Namely

$$
\tilde{Y}(t)=\int_{\mathbb{R}}\left(\frac{e^{i t \xi}-1}{|\xi|^{H(t)+\frac{1}{2}}}\right) \tilde{W}(d \xi) \quad \text { where } \quad H(t) \in(0,1)
$$

and where $\tilde{W}(d \xi)$ denotes a complex-valued Gaussian measure.

Observe that when $\mathrm{H}(\mathrm{t})=\mathrm{H}=$ const both $Y_{(0,1)}$ and $\tilde{Y}$ become fractional Brownian motion processes with selfsimilarity parameter $\mathrm{H}$. When $\mathrm{H}(\mathrm{t})$ is non constant but regular, then we expect that continuity, the finite-dimensional distribution of the MBM processes $\mathrm{Y}_{(0,1)}(\mathrm{t})$ and $\tilde{Y}(\mathrm{t})$ to be close to those of a FBM process $\mathrm{B}_{H\left(t_{0}\right)}(\mathrm{t})$, in a neighborhood of $\mathrm{t}_{0} \in \mathbb{R}$.

Indeed, Benassi and al have [1] shown, under some regularity conditions on the function $\mathrm{H}(\mathrm{t})$, that $\tilde{Y}(\mathrm{t})$ is locally asymptotically self-similar and that its tangent process is the
FBM process. For results about $\mathrm{Y}_{(0,1)}$ see for example, R.F Peltier and Levy-Vehel [14] and also Stoev, Taqqu and Al [5] for results about Gaussian and stable infinite variance processes.

R.F Peltier, Levy-Vehel and Benassi [14] have also shown that, under regularity conditions on the functions $\mathrm{H}(\mathrm{t})$, the paths of $Y_{(0,1)}$ and $\tilde{Y}$ have pointwise Holder exponent at $\mathrm{t}$, equal almost surely to $\mathrm{H}(\mathrm{t})$.

The processe $Y_{(0,1)}$ and $\tilde{Y}$ share many properties and it is important to establish their precise connection [8].

Serge Cohen [15] has shown the process $\tilde{Y}$ has the following time domain integral representation:

$$
\begin{aligned}
& \{\tilde{Y}(t)\}_{t \in \mathbb{R}}= \\
& \left\{K_{H}(t) \int_{\mathbb{R}}\left(|t-u|^{H(t)-\frac{1}{2}}-1-|-u|^{H(t)-\frac{1}{2}}\right) W(d u)\right\}
\end{aligned}
$$

where $K_{H}(t)$ is a determinisyic function and where the Kernel is

$$
\ln \left(\frac{1}{|t-u|}\right)-\ln \left(\frac{1}{|-u|}\right) \quad u \neq 0 \quad u \neq t \quad \text { if } \quad H(t)=\frac{1}{2} .
$$

Observe that the stochastic integrals in relations (2) and (4) involve different Kernel functions.

Here, we study the correlation structures of the MBM processes $Y_{(0,1)}$ and $\tilde{Y}$, extending results of Serge Cohen and Ayache [14].

In fact Stillian and Taqqu [5] shown that the MBM processes $Y_{(0,1)}$ and $\tilde{Y}$ have generally different correlation structures, when the function $\mathrm{H}(\mathrm{t})$ is non constant.

Definition 2.3. Let $h: \mathbb{R}^{d} \rightarrow(0,1)$ be a measuarable function. A real valued field is called a multifractional Brownian field with multifractional function $\mathrm{h}$, if it admits the harmonisable representation

$$
B_{h}(t)=\frac{1}{(C(h(x)))^{\frac{1}{2}}} \int_{\mathbb{R}^{d}} \frac{e^{-i x \xi}-1}{\|\xi\|^{\frac{d}{2}+h(x)}} \tilde{W}(d \xi)
$$

where the normalization function is

$$
C(s)=\int_{\mathbb{R}^{d}} \frac{2\left(1-\cos \left(\xi_{1}\right)\right)}{\|\xi\|^{d+2 s}} \frac{d \xi}{(2 \pi)^{\frac{d}{2}}}=\frac{\pi^{\frac{1}{2}} \Gamma\left(s+\frac{1}{2}\right)}{2^{\frac{d}{2}} \Gamma(2 s) \sin (\pi s) \Gamma\left(s+\frac{d}{2}\right)}
$$


If $\mathrm{d}=1$ multifractional Brownian fields $(\mathrm{mBf})$ are often called multifractional Brownian motion

\section{Some Fondamental Results}

Proposition 3.1. Let $B_{h}$ be an $\mathrm{mBm}$ with multifractional function h. then

$$
E\left(B_{h}(x) B_{h}(y)\right)=D(h(x), h(y))\left(\|x\|^{h(x)+h(y)}+\|y\|^{h(x)+h(y)}-\|x-y\|^{h(x)+h(y)}\right)
$$

where

$$
D(s, t)=\frac{\sqrt{\Gamma(2 s+1) \Gamma(2 t+1) \sin (\pi s) \sin (\pi t)} \Gamma\left(\frac{s+t+1}{2}\right)}{2 \Gamma(s+t+1) \sin \left(\frac{\pi(s+t)}{2}\right) \Gamma\left(\frac{s+t+d}{2}\right)} \quad s, t \in(0,1)
$$

Proof.

$$
\begin{aligned}
E\left(B_{h}(x) B_{h}(y)\right) & =E\left(\frac{1}{C(h(x))^{\frac{1}{2}}} \int_{R^{d}} \frac{e^{-i x \xi}-1}{\|\xi\|^{\frac{d}{2}+h(x)}} \tilde{W}(d \xi) \frac{1}{C(h(y))^{\frac{1}{2}}} \int_{\mathbb{R}^{d}} \frac{e^{i y \xi}-1}{\|\xi\|^{\frac{d}{2}+h(y)}} \tilde{W}(d \xi)\right) \\
& =\frac{1}{C(h(x))^{\frac{1}{2}} C(h(y))^{\frac{1}{2}}} \int_{\mathbb{R}^{d}} \frac{\left(e^{-i x \xi}-1\right)\left(e^{i y \xi}-1\right)}{\|\xi\|^{h(x)+h(y)+d}} \frac{d \xi}{(2 \pi)^{\frac{d}{2}}}
\end{aligned}
$$

Fix $x, y \in \mathbb{R}^{d}$, and let $B_{H}$ be a standard fractional Brownian field with fractional parameter $H=\frac{h(x)+h(y)}{2}$ because of the harmonizable representation of fractional Brownian field we know

$$
\begin{aligned}
E\left(B_{h}(x) B_{h}(y)\right) & =\frac{1}{C_{H}} \int_{\mathbb{R}^{d}} \frac{\left(e^{-i x \xi}-1\right)\left(e^{i y \xi}-1\right)}{\|\xi\|^{2 H+d}} \frac{d \xi}{(2 \pi)^{\frac{d}{2}}} \\
& =\frac{1}{2}\left(\|x\|^{2 H}+\|y\|^{2 H}-\|x-y\|^{2 H}\right) \int_{\mathbb{R}^{d}} \frac{\left(e^{-i x \xi}-1\right)\left(e^{i y \xi}-1\right)}{\|\xi\|^{h(x)+h(y)+d}} \frac{d \xi}{(2 \pi)^{\frac{d}{2}}} \\
& =\frac{C_{\frac{h(x)+h(y)}{2}}}{2}\left(\|x\|^{h(x)+h(y)}+\|y\|^{h(x)+h(y)}-\|x-y\|^{h(x)+h(y)}\right)
\end{aligned}
$$

Replacing by $\frac{h(x)+h(y)}{2}$ in $\mathrm{C}(\mathrm{s})$ and using the identity $x \Gamma(x)=\Gamma(x+1)$ yields the announced equality.[2]

Let us define

$$
\chi_{\lambda}(x, y)=\int_{\mathbb{R}} \frac{e^{-i x y}-1}{|\xi|^{y+\frac{1}{2}}} \overline{\Psi_{\lambda}(\xi)} \frac{d \xi}{(2 \pi)^{\frac{1}{2}}}
$$

In the proposition, we give another proof of the almost sure uniform convergence on a compact $\mathrm{K}$ of the series

$$
=\frac{1}{C(h(x))^{\frac{1}{2}}} \sum_{\lambda \in \Lambda^{+}} \chi_{\lambda}(t, h(t)) \eta_{\lambda} \quad \text { (5) with } \quad \eta_{\lambda}=H
$$

Proposition 3.2. Let h be function locally Hölder continuous with exponent $\beta$ then we show the following results.

1 - the uniform convergence of the series on a compact $\mathrm{K}$

$$
\sum_{j \geqslant J, k \in \mathbb{Z}} \chi_{j, k, 1}(t, h(t)) \eta_{j, k, 1} \longrightarrow 0 \quad \text { when } \quad J \rightarrow+\infty
$$

2- For $j>0$ the uniform convergence of the series on $\mathrm{K}$

$$
\sum_{|k| \geqslant K} \chi_{j, k, 1}(t, h(t)) \eta_{j, k, 1} \longrightarrow 0 \quad \text { when } K \rightarrow+\infty .
$$

3- The uniform convergence of the series on $\mathrm{K}$

$$
\sum_{j \geqslant J, k \in \mathbb{Z}} \tilde{\chi}_{0, k, 0}(t, h(t)) \eta_{0, k, 0} \longrightarrow 0 \quad \text { when } K \rightarrow+\infty .
$$


4- The uniform convergence of the series on $\mathrm{K}$

$$
\sum_{j \geqslant J, k \in \mathbb{Z}} \chi_{0, k, 0}(t, h(t)) \eta_{0, k, 0} \longrightarrow 0 \quad \text { when } \quad K \rightarrow+\infty .
$$

5- Deduce from the previous questions the almost sure uniform convergence of the series (5) to a $\mathrm{mBm}$.

1 - Since $\mathrm{h}$ is continuous on $\mathrm{K}$ the minimum $a=\inf \{h(t), t \in K\}$ is positive.

$$
\left|\sum_{j \geqslant J, k \in \mathbb{Z}} \chi_{j, k, 1}(t, h(t)) \eta_{j, k, 1}\right| \leqslant \sum_{j \geqslant J} 2^{-a j} \sum_{k \in \mathbb{Z}} \frac{\ln (1+j)^{\frac{1}{2}}+\ln (1+|k|)^{\frac{1}{2}}}{1+\left|2^{j} t-k\right|^{2}}+\frac{\ln (1+j)^{\frac{1}{2}}+\ln (1+|k|)^{\frac{1}{2}}}{1+|k|^{2}}
$$

and we get

$$
\left|\sum_{j \geqslant J, k \in \mathbb{Z}} \chi_{j, k, 1}(t, h(t)) \eta_{j, k, 1}\right| \leqslant C 2^{-a^{\prime} j}
$$

where $a^{\prime}<a$. It shows the almost sure uniform convergence on $\mathrm{K}$.

$2-$ As in the provious question

$$
\left|\sum_{|k| \geqslant K^{\prime}} \chi_{j, k, 1}(t, h(t)) \eta_{j, k, 1}\right| \leqslant C 2^{-a j} \leqslant \sum_{|k| \geqslant K^{\prime}} \frac{\ln (1+j)^{\frac{1}{2}}+\ln (1+|k|)^{\frac{1}{2}}}{1+\left|2^{j} t-k\right|^{2}}+\frac{\ln (1+j)^{\frac{1}{2}}+\ln (1+|k|)^{\frac{1}{2}}}{1+|k|^{2}}
$$

Let us show the almost sure uniform convergence on $\mathrm{K}$ of $\sum_{|k| \geqslant K^{\prime}} \frac{1}{1+\left|2^{j} t-k\right|^{2}}$. Let us denote by

$$
k^{\prime}\left(K^{\prime}\right)=\inf \left\{\left|2^{j} t-k\right|^{2}, \quad \forall t \in K, \quad \forall k \in \mathbb{Z}, \quad|k| \geqslant K^{\prime}\right\}
$$

and let us remark that $\lim _{K^{\prime} \rightarrow} k^{\prime}\left(K^{\prime}\right)=\infty$ since $\mathrm{K}$ is compact, Hence

$$
\sum_{|k| \geqslant K^{\prime}} \frac{1}{1+\left|2^{j} t-k\right|^{2}} \leqslant C \sum_{|k| \geqslant k^{\prime}\left(K^{\prime}\right)} \frac{1}{k^{2}} \rightarrow 0 \text { when } K^{\prime} \rightarrow+\infty
$$

The uniform convergence on $\mathrm{K}$ of $\sum_{|k| \geqslant K} \chi_{j, k, 1}(t, h(t)) \eta_{j, k, 1}$ is a consequence of the previous fact. $3-$

$$
\left|\sum_{|k| \geqslant K^{\prime}} \tilde{\chi}_{0, k, 0}(t, h(t)) \eta_{0, k, 0}\right| \leqslant C \sum_{|k| \geqslant K^{\prime}}\left(\frac{1}{1+|t-k|^{2}}+\frac{1}{1+|k|^{2}}\right) \rightarrow\left|\eta_{0, k, 0}\right|
$$

which almost surely uniformly convergence on $\mathrm{K}$ by similar arguments as the one we used in the previous question.

4- Let us consider

$$
\begin{gathered}
M_{K^{\prime}}(t)=\sum_{0<k<K^{\prime}} \tilde{\chi}_{0, k, 0}(t, h(t))-\chi_{0, k, 0}(t, h(t)) \eta_{0, k, 0} \\
M_{K^{\prime}}(t)=E\left(C \int_{|\xi| \leqslant \frac{4}{3} \pi} \frac{i t \xi-\frac{1}{2 t^{2}} \xi^{2}}{|\xi|^{\frac{1}{2}+h(t)}} \tilde{W}^{+}(d \xi) / \tau_{K^{\prime}}\right) \quad \text { where } \tau_{K^{\prime}}=\sigma\left(\eta_{0, k, 0}, 0 \leqslant k \leqslant k^{\prime}\right)
\end{gathered}
$$

Morever the process

$$
\chi(t, h(t))=\int_{|\xi| \leqslant \frac{4}{3} \pi} \frac{i t \xi-\frac{1}{2 t^{2}} \xi^{2}}{|\xi|^{\frac{1}{2}+h(t)}} \tilde{W}^{+}(d \xi) \text { is almost surely continuous. }
$$

$M_{K}$ converges almost surely uniformly on every compact. The same property is obviously true for

$$
\sum_{0<k<k^{\prime}}\left(\tilde{\chi}_{0, k, 0}(t, h(t))-\chi_{0, k, 0}(t, h(t))_{\eta_{0, k, 0}}\right)
$$

Hence we get the result.

5- Since the normalization function is analytic and non vanishing, and $\mathrm{h}$ is continuous $\frac{1}{C(h(x))^{\frac{1}{2}}}$ is bounded on $\mathrm{K}$, and the result is a consequence of 1,2 and 4 . 
Proposition 3.3. Let $h: \mathbb{R}^{d} \rightarrow(0,1)$ be a $\beta$-Hölder continuous multifractional function and $B_{h}$ be the corresponding multifractional Brownian field let us assume $\beta>\sup _{x \in \mathbb{R}^{d}} h(x)$, then, for every $x \in \mathbb{R}^{d}$ the $\mathrm{mBf}$ is strongly locally asymptotically self-similar, with tangent field a fractional Brownian field with Hurst exponent $\mathrm{H}=\mathrm{h}(\mathrm{x})$. More precisely

$$
\lim _{\varepsilon \rightarrow 0^{+}}\left(\frac{B_{h}(x+\varepsilon u)-B_{h}(x)}{2^{h(x)}}\right)={ }^{d} B_{H}(u)_{u \in \mathbb{R}^{d}}
$$

Proof. Let us fix $x \in \mathbb{R}^{d}$, for every $u_{1}, u_{2} \in \mathbb{R}^{d}$

$$
\begin{aligned}
\frac{E\left(B_{h}\left(x+\varepsilon u_{1}\right)-B_{h}(x)\right)\left(B_{h}\left(x+\varepsilon u_{2}\right)-B_{h}(x)\right)}{\varepsilon^{2 h(x)}} & =\frac{1}{2 \varepsilon^{2 h(x)}} \times E\left(B_{h}\left(x+\varepsilon u_{1}\right)-B_{h}(x)\right)^{2} \\
& +E\left(B_{h}\left(x+\varepsilon u_{2}\right)-B_{h}(x)\right)^{2} \\
& -E\left(B_{h}\left(x+\varepsilon u_{2}\right)-B_{h}\left(x+\varepsilon u_{1}\right)\right)^{2}
\end{aligned}
$$

Hence,

$$
\left(\lim _{\varepsilon \rightarrow 0^{+}} \frac{E\left(B_{h}\left(x+\varepsilon u_{1}\right)-B_{h}(x)\right)\left(B_{h}\left(x+\varepsilon u_{2}\right)-B_{h}(x)\right)}{\varepsilon^{2 h(x)}}\right)=\frac{1}{2}\left(\left\|u_{1}\right\|^{2 h(x)}+\left\|u_{2}\right\|^{2 h(x)}-\left\|u_{1}-u_{2}\right\|^{2 h(x)}\right)
$$

which yields the convergence of finite dimensional margins then, to have the convergence in distribution in the space of continuous functions endowed with the topology of the uniform convergence on every compact, we have to prove the lightness of the renormalized increments.

$$
Y_{\varepsilon}(u)=\frac{B_{h}(x+\varepsilon u)-B_{h}(x)}{\varepsilon^{h(x)}}
$$

Let us fix a compact $\mathrm{K}$ then

$$
\lim _{\varepsilon \rightarrow 0} \frac{E\left(Y_{\varepsilon}\left(u_{1}\right)-Y_{\varepsilon}\left(u_{2}\right)\right)^{2}}{\left\|u_{1}-u_{2}\right\|^{2 h(x)}}=1
$$

Uniformly when $u_{1}, u_{2}$ are in the compact $\mathrm{K}$, hence

$$
\sup _{\varepsilon>0} E\left(Y_{\varepsilon}\left(u_{1}\right)-Y_{\varepsilon}\left(u_{2}\right)\right)^{2} \leqslant C\left\|u_{1}-u_{2}\right\|^{2 h(x)}
$$

then, one can choose $p \in \mathbb{N}$ such that $p h(x)>d$ since

$$
E\left(Y_{\varepsilon}\left(u_{1}\right)-Y_{\varepsilon}\left(u_{2}\right)\right)^{2 p} \leqslant C\left\|u_{1}-u_{2}\right\|^{p h(x)}
$$

\section{Conclusion and Comments}

After having determined the covariance function, we give in proposition 2 another proof of almost sure uniform convergence on compact $\mathrm{K}$ of the series.

We will finish by showing that the m.B.f is locally asymptotically self-similar, with field or fractional Brownian field with Hurst exposant $H=h(x)$.

Actually if we consider the series expansion of the $\mathrm{mBm}$ and consider only a finite number of terms in

$$
\sum_{\lambda \in \Lambda} \chi_{\lambda}(t, h(t)) \eta_{\lambda}
$$

The sum process cannot be in general more regular than h. On the other hand the Hölder exponent of the sample path of the $\mathrm{mBm}$ is related to the behavior of $\chi_{\lambda}(x, y)$ when the frequency part $\mathrm{j}$ of $\lambda=(j, k)$ tends to infinity. So, in some sense, the obtruction to have an irregular multifractional function is a low frequency problem [3]. These considerations lead to propose a generalized model of the $\mathrm{mBm}$, where the multifractional function $\mathrm{h}(\mathrm{t})$ may depend of the frequency and, this becomes a function $(t, \xi) \rightarrow H(t, \xi)$ [10] and [6].

Let us discurs more precisely assumptions required for this function.

Hypothesis A function $H: R \times R \rightarrow[a, b] \subset[0,1]$ is called a frequency lift of a multifractional function $h n$ if

$$
\begin{gathered}
h(t)=\lim _{\xi \rightarrow+\infty} H(t, \xi) \\
H(t,-\xi)=H(t, \xi)
\end{gathered}
$$

$\mathrm{H}$ is twice differentiable with aspect to the variable $\xi$ and satisfies

(1) for $0<|\xi| \leqslant \frac{4 \pi}{3} H(t, \xi)=b$

(2) for $\frac{4 \pi}{3}<|\xi|, \exists \mathrm{g}$ such that $0<g<\min \left(\frac{a}{2}, \frac{1}{4}\right)$ and $\exists \beta$ such that $b<\beta \leqslant 1$

$$
\left|\frac{\partial^{k} H}{\partial \xi^{k}}(t, \xi)-\frac{\partial^{k} H}{\partial \xi^{k}}\left(t^{\prime}, \xi\right)\right| \leqslant C|\xi|^{g-k}\left|t-t^{\prime}\right|^{\beta}
$$

for $\mathrm{t}, t^{\prime} \in[0,1]$ and $\mathrm{k}=0,1,2$

$$
\left|\frac{\partial^{k} H}{\partial \xi^{k}}(t, \xi)\right| \leqslant C|\xi|^{g-k}
$$

for $t \in[0,1]$ and $\mathrm{k}=0,1,2$.

These assumptions, that are needed to construct an Holder continuous model, are quite technical.

If one wishes a model similar to $\mathrm{mBm}$ but for an irregular multifractional functions $h$, we have to construct a frequency lift of the desired multifractional function $\mathrm{h}$ [13].

In Hypothesis the cut-off at $\xi=\frac{4 \pi}{3}$ is arbitrary and be replaced by any non - negative constant. The inequality $0<$ $g<\min \left(\frac{a}{2}, \frac{1}{4}\right)$ has a deeper meaning : it expresses the fact that the Hölder constant of $H(t, \xi)$ cannot grow too fast when $\xi \rightarrow+\infty$. Finally, the inequality $b<\beta$ is similar to the conditions $\beta>\sup _{x \in R^{d}} h(x)$ in prop 3 . 


\section{References}

[1] A. Ayache, A. Benassi, S. Cohen, J. LevyVehel: Regularity and Identification of Generalized Multifractional Gaussian Processes, in: Lecture Notes in Math, vol 1857 Spinger, Berlin, 2005, pp 290-312.

[2] A. Ayache, S. Cohen, J. L. Vehel the covariance structure of multifractional Brownian motion in : IEEE ICASSP 2000 .

[3] A. Begyn, Asymptotic expansion and central limit theorem for quadratic variation of Gaussian processes, stochastic Process. Appl 117 (2007).

[4] A. Brouste J. Ista and S. Lambert- Lacroix On fractional Gaussian random fields simulation J. Stat. Soft 2007.

[5] A. Stoev Stilian, Murad S. Taqqu: How rich in the class multifractional brownian motion, stochastic Processes and their applications 116, 2006, 200-221.

[6] A. Benassi, S Jaffard, D. Roux, Elliptic Gaussian fandom processes, rev. Math Iber 13 (1) 1997 19-90.

[7] Ba Demba Bocar: On the fractional Brownien motion: Hausdorf dimension and Fourier expansion international

journal of advances in applied mathematical and mechanics vol 5 pp 53-59 (2017).

[8] Ba Demba Bocar: Fractional operators and Applications to fractional martingal international journal of advances in applied mathematical and mechanics vol 5 (2018).

[9] C. Lacaux Real harmonizable multifractional Levy motions. Ann. Inst. Poincarre Proba-Stat 2004.

[10] C. Locaux Fields with exceptional tangent fields. J. theorie Proba 2005.

[11] Mandelbrot and J. W.Van Ness, Fractional Brownian motion, fractional noises and applications STAN Rev, 10 $422-437,1968$.

[12] M. Clausel . Lacunary fractional Brownian motion E SAIM. 2012.

[13] P. K. Frizand N. B. Victor, Multidimensional Stochastic Processes as Rough Paths: theory and Applications. Cambridge University Press, 2010.

[14] R. F Peltier, J. L. Vehel, Multifractional Brownian motion, definition and preliminary results, technical Report 2645, INRIA, le chesnay France, 1995.

[15] Serge Cohen, Jacques Istas, Fractional fields and applications. Springer 2010. 